\title{
Effects of Low-Level Laser Therapy as an Adjunct for Orthodontic Retention: A Systematic Review of Human and Animal Studies
}

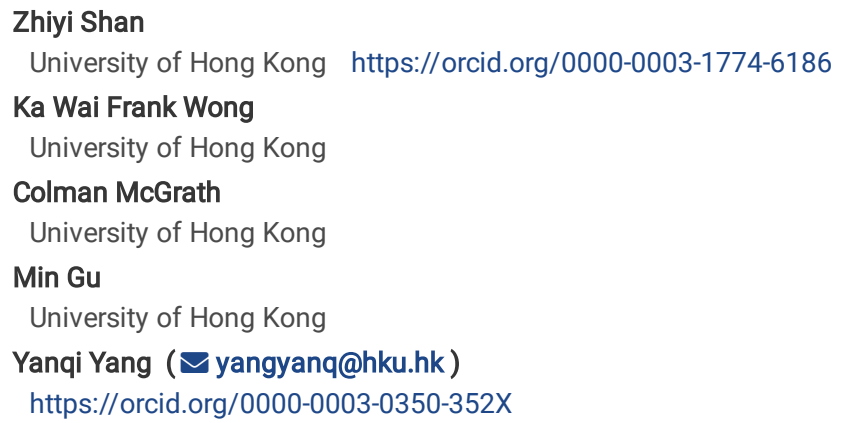

Keywords: low-level laser therapy, systematic review, orthodontic retention, orthodontically induced inflammatory root resorption

Posted Date: May 15th, 2020

DOI: https://doi.org/10.21203/rs.3.rs-28836/v1

License: (c) (7) This work is licensed under a Creative Commons Attribution 4.0 International License. Read Full License 


\section{Abstract}

Background: Low-level laser therapy (LLLT) has been verified effective in tooth-movement acceleration and pain alleviation during active orthodontic treatment, but its function remains inconclusive post-treatment. This systematic review aims to evaluate the effects of LLLT as an adjunct retention regimen following active orthodontic tooth movement (OTM).

Methods: This review was conducted according to the Preferred Reporting Items for Systematic Reviews and Meta-Analyses guidelines. Six databases (Cochrane Central Register of Controlled Trials, MEDLINE, Embase, Pubmed, Scopus, ProQuest) were comprehensively searched for human and animal studies published till December 2019 and screened according to our eligibility criteria. The risk of bias was assessed based on the Cochrane Handbook for Systematic Reviews of Interventions and Systematic Review Center for Laboratory Experiment Tool. Two independent reviewers performed all procedures in duplicate. Any disagreement was resolved by discussion or consultation with a third reviewer.

Results: A total of 394 records were identified from the initial search. Following screening, 15 full-text articles were reviewed for eligibility ( $\mathrm{k}>0.90)$, and ultimately, eight studies (three human studies and five animal studies) were included in this review. The key outcomes considered were 'preventing tooth relapse' and 'rehabilitating root resorption'. Two controlled clinical trials (CCTs) and one animal study supported the preventive effects of LLLT on the relapse of post-orthodontic tooth positions. In contrast, two animal studies reported opposing findings. Regarding the rehabilitation of root resorption, evidence supported the reparative potential of LLLT in orthodontic force-induced root resorption. Overall, there was a high risk of bias among studies, except for one randomised controlled trial. Due to the substantial heterogeneity among studies in terms of their types, participants, designs, LLLT settings and variables of interest, it was not feasible to conduct a meta-analysis; therefore, a qualitative synthesis is presented.

Conclusion: The quality of evidence for LLLT contributing to the maintenance of orthodontic outcomes or a better treatment prognosis remains low. There is considerable controversy over the effects of LLLT on orthodontic relapse. However, the use of LLLT after OTM has promising reparative effects for root resorption and is generally recommended.

\section{Background}

Maintaining the stability of satisfactory results achieved by orthodontic treatment is of great importance and expectation for both patients and clinicians. Harmonious occlusions and some degree of over-correction is recommended at the finishing stage, and long-term administration of retainers, such as Hawley's, lingual-fixed appliances or vacuum-formed retainers, is widely accepted as a clinical routine to maintain the results acquired by orthodontic tooth movement (OTM). Despite these conventional regimens, orthodontic relapse, defined as immediate or postponed drifting of teeth towards their original sites, is still inevitable in clinical practice [1,2]. This is especially true for teeth with initial rotation or with compromised periodontal supports. Strategies have been proposed to supplement the conventional retention regimen, including circumferential supracrestal fiberotomy [3], local injection of biological reagents [4-6] and systematic prescription of some cardiovascular medications [7, 8]. However, these approaches have met with limited success and have been widely criticized for their drawbacks such as the introduction of invasive wounds, uncertain efficacy for the suffers, and potential systematic side effects [9].

One main reason for the difficulty in preventing orthodontic relapse may be the cellular and molecular changes underlying orthodontically repositioned teeth. Previous views on relapse suggest that it is caused by the release of mechanical force stored in deformed collagen fibres at supra-alveolar areas during OTM [10-12]. In the late 1990s, Redlich et al. [13] established that the changes in the elastic properties of gingival tissues are the main cause of relapse, rather than collagen fibres. Several subsequent studies also highlighted the role of hard tissues in post-orthodontic instability and suggested that the remodelling of all surrounding tissues contributes to orthodontic relapse: From the perspective of soft tissues, collagen fibres are documented to influence short-term relapse, whereas elastic fibres contribute more to long-term mobility because they have extensive crosslinks with each other and are the slowest to degrade [14, 15]; Regarding hard tissues, researchers found that adjacent alveolar bones undergo a similar procedure between relapse and active treatment [16, 17] when osteogenesis and osteoclastogenesis both significantly participate in tooth resettlement. Accordingly, manipulating the metabolism of adjacent hard and soft tissues appears to be a promising approach to alleviate or even prevent orthodontic relapse.

Low-level-laser therapy (LLLT) is an adjunctive, non-invasive, highly compatible medical treatment for multiple abnormalities in dental field including aphthous stomatitis, periodontal disease, dental hypersensitivity, and orofacial pains [18-22]. The mechanism underpinning LLLT is the effect of photobiomodulation (PBM), which indicates regulating biological metabolic activities with photons. Fibroblasts from gingival and periodontal ligaments were reported to upregulate their anabolic activities and expression of mediators, such as heat shock proteins, transforming growth factor $\beta$, $\beta$-defensin 2 and basic fibroblast growth factor, after LLLT irradiation [23-25]. On the histological level, LLLT irradiated teeth showed a significant reduction in the coverage of non-epithelium gingival surfaces with less inflammation after gingivectomy [26]. With regard to hard tissues, osteoblastic-like cells are also reported to be susceptible to PBM and to increase their potential for proliferation, adhesion, differentiation and mineralisation under specific settings [27-30]. Several clinical studies have documented that LLLT could enhance the stability of bone-anchored mini-screws during active loading [31-33] and could also accelerate bone regeneration at enlarged midpalatal sutures while expanding the maxillary width [34-36] because of a higher mineral apposition rate in laser-irradiated areas as revealed by radiographic examination. Therefore, it appears that conceptually LLLT could be a promising adjunct to the conventional retention regimen and compensate for the post-OTM risks by modulating bone and soft tissue metabolism.

Recently, the application of LLLT in the orthodontic field has been verified effective in OTM acceleration and pain alleviation [37-40], but it remains inconclusive as to whether LLLT impacts the post-OTM status, including aspects of orthodontic relapse, dental or periodontal health and patients' selfperception. One previous systematic review surveyed only clinical trials investigating the effects of LLLT on orthodontic relapse and did not include any eligible studies with more than 20 subjects in each group [41]. Another systematic review synthesised the evidence from both human and animal studies but only considered the effects on rotational relapse instead of all relapse variations [42]. This systematic review was conducted to weigh positive and negative 
factors for the application of LLLT after the removal of active orthodontic forces by analysing current evidence of the effects of LLLT in post-OTM treatments, especially in terms of orthodontic relapse, dental or periodontal health and patients' self-perception.

\section{Question specification formatted in PICOS}

Population: Subjects who have undergone active OTM

Intervention: LLLT

Comparison: Placebo

Outcomes: Primary outcomes (post-OTM tooth stability, dental or periodontal health status and participant's subjective perceptions); secondary outcomes (histological or biochemical changes)

Study designs: Randomized-controlled trial (RCTs), clinical-controlled trials (CCTs), and animal experiments

\section{Methods}

This systematic review was performed and reported following the instructions of the Preferred Reporting Items for Systematic Reviews and Meta-Analyses guideline $[43,44]$. The protocol has been prospectively registered on the PROSPERO online database (CRD49019132133).

\section{Search strategy}

Two reviewers (ZYS and FW) independently conducted an electronic systematic search of six major databases, namely Cochrane Central Register of Controlled Trials, MEDLINE (via Ovid 1946), Embase (via Ovid 1974), Pubmed (1997), Scopus and ProQuest, for articles published up to March 2020, with the language restricted to English. Clinicaltrails.gov was also considered under our scope to avoid omissions of ongoing clinical studies. Medical Subject Headings, free text words and their synonyms were applied as search terms, including 'orthodontic/appliance/force', 'retention/maintenance/stability/relapse' and 'low-level laser/low-intensity laser/soft laser/photomodulation'. The detailed search strategy is presented in Appendix 1.

\section{Study selection}

The eligibility criteria are listed as follows.

Inclusion criteria

1. Randomised controlled trials (RCTs) or controlled clinical trials (CCTs) involving patients with and without irradiation by low-level lasers after active orthodontic treatment.

2. Experimental animal studies involving subjects with and without low-level laser application following the termination of an active orthodontic $f$

Exclusion criteria

1. Studies with no subjects having received LLLT after active orthodontic treatment completion.

2. Studies involving subjects who have severe maxillofacial deformities or have undergone any orthopaedic or surgical procedures.

3. Studies involving subjects with systematic diseases or under any pharmaceutical therapies that could potentially affect bone and soft tissue remodelling.

4. In-vitro studies, case reports, reviews, personal opinions and technique description articles without sample reporting.

5. Human or animal studies without any demonstrations of post-OTM effects.

Accordingly, all titles and abstracts obtained from the electronic search were screened by the two reviewers independently. Full articles were retrieved for the final assessment and their reference lists were also screened based on the aforementioned criteria. During the process, any disagreement between the two reviewers was resolved by discussion or consultation with a highly experienced reviewer (YQY). Cohen's Kappa values were computed to verify the two-round inter-reviewer reliabilities, which would be considered acceptable if not lower than 0.6 .

\section{Data extraction and analysis}

The following data were extracted: general information (first author and year of publication), study type and design, participants and target teeth (sample size and characteristics), orthodontic regimen (active and post-active strategy and period), low-level laser protocols (types, wavelength, beam size, mode, output power, dosage density, time of onset, duration, frequency and method of delivery), assessments (approach, region of interest, outcome variables and timepoints); primary outcomes related to post-OTM tooth stability, dental or periodontal health status and participant's subjective perceptions; and secondary outcomes related to histological or biochemical changes. 


\section{Assessment the risk of bias}

The assessment for the risk of bias of all human studies was performed in RevMan5.3 [45] using the Cochrane Risk of Bias Tool [46]. Seven domains were considered: (1) random sequence generation; (2) allocation concealment; (3) blinding of participants and personnel; (4) blinding of outcome assessment; (5) incomplete outcome data; (6) selective reporting; and (7) other bias. For the animal studies, the risk of bias was assessed based on the Systematic Review Center for Laboratory Experiment Tool (SYRCLE tool) [47]. Ten domains covering the aspects of selection, performance, detection, attrition and reporting were considered to grade the quality of evidence.

\section{Data synthesis}

The data of interest from human and animal studies were synthesised separately because of the substantial differences between them. Within each study type, data on individual settings, eligibility criteria, study design, intervention, outcome variables and statistical methods were further analysed. If heterogeneity was acceptable, quantitative synthesis and meta-analysis of the retrieved data were performed; otherwise, a narrative description is presented.

\section{Results}

\section{Characteristics of selected studies}

The process of study search and selection is illustrated in Fig. 1. The electronic search until March 2020 yielded a total of 386 relevant records from the six databases; another eight records were identified by screening bibliographies. After removing duplicates, the remaining 298 studies were analysed by title and abstract, which left 15 articles for full-text comprehension. According to the eligibility criteria, seven of these articles were further excluded for specific reasons. Finally, this systematic review included three clinical trials [48-50] and five animal studies [51-55]. Cohen's kappa coefficient values were 0.82 and 0.78 for the above two selection rounds, indicating a substantial agreement between the two reviewers.

Only tooth relapse and dental health in terms of root resorption were investigated out of all post-OTM outcomes, and no studies reported any impacts of LLLT on patients' subjective perceptions. For orthodontic relapse, two of the studies were CCTs [48, 49] and the others were animal experiments [51-53]. These studies covered two types of tooth movement, rotational and transitional relapse. The other prognosis was orthodontically induced inflammatory root resorption (OIIRR), which was reported in one RCT [50] and two animal studies [54, 55]. Considering the substantial heterogeneity exhibited in outcomes, no meta-analysis could be performed; therefore, a qualitative synthesis of LLLT's post-OTM effects was performed in a narrative manner.

\section{Effects on post-OTM stability of tooth position}

Two CCTs $[48,49]$ and three animal studies [51-53] fulfilled the inclusive criteria investigating the effects of LLLT on post-OTM stability of tooth position, and the results they documented were inconclusive. Detailed information from all studies on post-OTM stability is summarised in Table 1. 


\begin{tabular}{|c|c|c|c|c|c|c|c|c|c|}
\hline \multirow{2}{*}{$\begin{array}{l}\text { Study } \\
\text { type }\end{array}$} & \multirow{2}{*}{$\begin{array}{l}\text { Authors } \\
\text { and year }\end{array}$} & \multirow{2}{*}{$\begin{array}{l}\text { Study } \\
\text { design }\end{array}$} & \multirow{2}{*}{$\begin{array}{l}\text { Participants } \\
\text { and teeth }\end{array}$} & \multicolumn{3}{|l|}{ Interventions } & \multicolumn{3}{|l|}{ Assessment } \\
\hline & & & & $\begin{array}{l}\text { Active } \\
\text { treatment } \\
\text { strategy and } \\
\text { period }\end{array}$ & $\begin{array}{l}\text { Post- } \\
\text { orthodontic } \\
\text { strategy }\end{array}$ & $\begin{array}{l}\text { Observation } \\
\text { time }\end{array}$ & Measure method & $\begin{array}{l}\text { Targeted } \\
\text { regions }\end{array}$ & $\begin{array}{l}\text { Outcome } \\
\text { variables }\end{array}$ \\
\hline \multirow{6}{*}{$\begin{array}{l}\text { Clinical } \\
\text { studies }\end{array}$} & \multirow{5}{*}{$\begin{array}{l}\text { Zahra et } \\
\text { al (2009) } \\
{[48]}\end{array}$} & \multirow{5}{*}{$\begin{array}{l}\mathrm{CCT}, \\
\text { parallel }\end{array}$} & \multirow{5}{*}{$\begin{array}{l}\mathrm{N}=14 \\
(\mathrm{Nm}=3, \\
\mathrm{Nf}=11) \\
\text { aged 19- } \\
27 y\end{array}$} & \multirow{5}{*}{$\begin{array}{l}\text { FA (Roth } \\
\text { prescription); } \\
\text { 8-16m }\end{array}$} & 45d FA; & $\otimes 15 d$ & \multirow{5}{*}{$\begin{array}{l}\text { Direct measurement } \\
\text { on study model }\end{array}$} & \multirow{5}{*}{$\begin{array}{l}\text { Incisal region } \\
\text { and adjacent } \\
\text { alveolar } \\
\text { bones }\end{array}$} & \multirow{5}{*}{$\begin{array}{l}\otimes \text { Diastema } \\
\text { size } \\
\otimes \text { Changes of } \\
\text { alveolar bone } \\
\text { density and } \\
\text { height }\end{array}$} \\
\hline & & & & & \multirow{4}{*}{$\begin{array}{l}6 \mathrm{~m} \text { Hawley } \\
\text { retainer }\end{array}$} & $\nabla 45 d$ & & & \\
\hline & & & & & & $\nabla 3 \mathrm{~m}$ & & & \\
\hline & & & & & & $\nabla 6 \mathrm{~m}$ & & & \\
\hline & & & & & & $\nabla 1.5 \mathrm{y}$ & & & \\
\hline & $\begin{array}{l}\text { Jahanbin } \\
\text { et al } \\
(2014) \\
{[49]}\end{array}$ & $\begin{array}{l}\mathrm{CCT}, \\
\text { parallel }\end{array}$ & $\begin{array}{l}\mathrm{N}=24 \\
(\mathrm{Nm}=4, \\
\mathrm{Nf}=20) \\
\text { aged 16- } \\
32 \mathrm{y} ; \\
\text { Subjects: } \\
\mathrm{n}=47 \text { teeth }\end{array}$ & $\mathrm{FA} ; \mathrm{N} / \mathrm{A}$ & $\begin{array}{l}\text { No } \\
\text { retention }\end{array}$ & $1 \mathrm{~m}$ & $\begin{array}{l}\otimes \text { Computer } \\
\text { measurements on } \\
\text { standardized photos }\end{array}$ & $\begin{array}{l}\text { Pretreatment- } \\
\text { rotated } \\
\text { incisors }\end{array}$ & $\begin{array}{l}\text { D percentage } \\
\text { of relapse }\end{array}$ \\
\hline \multirow[t]{6}{*}{$\begin{array}{l}\text { Animal } \\
\text { studies }\end{array}$} & \multirow{4}{*}{$\begin{array}{l}\text { Kim et al } \\
(2010) \\
{[51]}\end{array}$} & \multirow{4}{*}{$\begin{array}{l}\text { Dogs } \\
(\mathrm{N} / \mathrm{A}) \\
\text { parallel }\end{array}$} & \multirow[t]{4}{*}{$\mathrm{N}=9, \mathrm{n}=18$} & \multirow{4}{*}{$\begin{array}{l}\text { Rotational } \\
\text { couple force } \\
\text { (elastic } \\
\text { chains } \\
\text { engaged } \\
\text { between } \\
\text { buttons for } \\
50 \mathrm{~g}) ; 4 \mathrm{w}\end{array}$} & \multirow[t]{4}{*}{$\begin{array}{l}\text { No } \\
\text { retention }\end{array}$} & \multirow[t]{4}{*}{4 weeks } & \multirow{4}{*}{$\begin{array}{l}\bigotimes \text { Computer } \\
\text { measurements on } \\
\text { standardized photos } \\
\text { of study models }\end{array}$} & \multirow{4}{*}{$\begin{array}{l}\text { mandibular } \\
\text { lateral } \\
\text { incisors }\end{array}$} & $\begin{array}{l}\bigotimes \text { amount of } \\
\text { relapse }\end{array}$ \\
\hline & & & & & & & & & $\begin{array}{l}\square \text { sulcus } \\
\text { depth; }\end{array}$ \\
\hline & & & & & & & & & $\begin{array}{l}\bigotimes \text { gingival } \\
\text { recession; }\end{array}$ \\
\hline & & & & & & & & & $\begin{array}{l}\bigotimes \text { connective } \\
\text { tissue } \\
\text { rearrangement }\end{array}$ \\
\hline & \multirow{2}{*}{$\begin{array}{l}\text { Franzen } \\
\text { et al } \\
(2015) \\
{[52]}\end{array}$} & \multirow[t]{2}{*}{$\begin{array}{l}\text { Rats } \\
\text { parallel }\end{array}$} & \multirow[t]{2}{*}{$\mathrm{N}=61, \mathrm{n}=61$} & \multirow{2}{*}{$\begin{array}{l}\text { Mesial } \\
\text { displacement } \\
\text { force } \\
\text { (chrome } \\
\text { alloy closed } \\
\text { coil spring } \\
\text { for } 0.5 \mathrm{~N} \text { ); } 10 \\
\text { d }\end{array}$} & \multirow[t]{2}{*}{$\begin{array}{l}\text { No } \\
\text { retention }\end{array}$} & \multirow[t]{2}{*}{$\begin{array}{l}\text { Day } 1,3,5 \text {, } \\
7,14 \text {, and } \\
21\end{array}$} & $\begin{array}{l}\text { Direct measurement } \\
\text { using feeler gauge; }\end{array}$ & \multirow[t]{2}{*}{$\begin{array}{l}\text { maxillary } \\
\text { right first } \\
\text { molars }\end{array}$} & \multirow{2}{*}{ 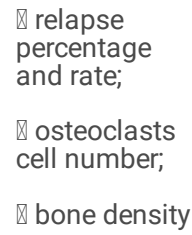 } \\
\hline & & & & & & & $\begin{array}{l}\text { Densitometric } \\
\text { analysis; Histological } \\
\text { analysis }\end{array}$ & & \\
\hline
\end{tabular}

\begin{tabular}{|c|c|c|c|c|c|c|c|c|}
\hline $\begin{array}{l}\text { Lee et al. } \\
(2016) \\
{[53]}\end{array}$ & $\begin{array}{l}\text { Rats } \\
\text { parallel }\end{array}$ & $\mathrm{N}=24$ & $\begin{array}{l}\text { Space-open } \\
\text { force (elastic } \\
\text { ring inserted }\end{array}$ & $\begin{array}{l}1 \mathrm{w} \\
\text { retention }\end{array}$ & $\begin{array}{l}\text { Day } 8,10 \\
\text { and } 13\end{array}$ & $\begin{array}{l}\text { Q Measurement on } \\
\text { study model; }\end{array}$ & $\begin{array}{l}\text { maxillary } \\
\text { central } \\
\text { incisors }\end{array}$ & $\nabla$ relapse rate; \\
\hline
\end{tabular}




$\begin{array}{lll}\text { between the } & \text { Real-time RT-PCR; } & \text { relative } \\ \text { maxillary } & & \text { mRNA } \\ \text { central } & \mathbb{1 m} & \text { translation } \\ \text { incisors); } 14 & \text { anmunohistochemistry } & \text { and protein } \\ \text { d (reactivate } & & \text { expression of } \\ \text { at day 7) } & \text { MMP-1, MMP- } \\ & 8, \text { MMP-13 } \\ & \text { and MMP-2, } \\ & \text { MMP-9; }\end{array}$

CCT: clinical controlled trial; SMD: split-mouth design; N: number of participants; $n$ : number of evaluated teeth; FA: fixed appliances; CSF: circumferential supr metalloproteinase; LLLT: low-level laser therapy

Table 1

Characteristics of included studies on orthodontic relapse

\section{Clinical studies}

The favourable preventive effects of LLLT on orthodontic relapse were reported in both CCTs, but one showed a statistically significant difference between groups [49] and one did not [48]. One study showed that teeth irradiated with a low-level laser (GaAlAs, $810 \mathrm{~nm}$, continuous wave, $\left.35.7 \mathrm{~J} / \mathrm{cm}^{2}\right)$ had a nearly $60 \%$ decrease in the amount of post-OTM relapse compared with their control counterparts $(p<0.05)[49]$. The other study using a low-level laser $(G a A s$, $904 \mathrm{~nm}$, pulse wave, $4.9 \mathrm{~J} / \mathrm{cm}^{2}$ ) detected a favourable reduction in orthodontic relapse but it did not reach statistical significance, except a significant increase in the alveolar crest height and a substantial retardation of bone density reduction in areas under irradiation were found [48].

Although the two studies consistently reported LLLT's positive effects on orthodontic relapse, their methodologies appeared to diverge. First, two types of postactive tooth movement were discussed. One study observed transitional tooth movement [48], whereas the other study examined the impacts on rotational relapse [49]. Second, the two studies adopted different retention regimens. One left the fixed appliances attached for the first 45 days and then used Hawley's retainers for the following 6 months [48], whereas the other did not use any post-OTM retainers [49]. Third, the adopted LLLT parameters were quite different. One study used irradiation with a GaAl laser at $904 \mathrm{~nm}$ in pulse waves and a 4.9-J/ $\mathrm{cm}^{2}$ dosage density [48], whereas the other applied a GaAlAs laser at $810 \mathrm{~nm}$ in continuous mode and a $35.7-\mathrm{J} / \mathrm{cm}^{2}$ dosage density [49]. Importantly, the assessment timepoints were also not comparable. One study undertook a long-term observation for 1.5 years [48], whereas the other had an observation period of 30 days post-OTM [49].

\section{Animal studies}

The animal studies investigating the effects of LLLT on post-OTM tooth stability include one that assessed rotation [51] and two that observed transitional movement $[52,53]$ in rodent and canine models, respectively.

For rotational relapse, Kim et al. [51] found that a GaAlAs laser ( $808 \mathrm{~nm}$, pulse wave, $\left.4.63-6.47 \mathrm{~J} / \mathrm{cm}^{2}\right)$ deteriorated post-OTM stability of tooth position by approximately $15 \%$ when not using any retainers $(p<0.05)$. For transitional relapse, one rodent study showed a positive effect of LLLT on tooth stability (GaAlAs, $830 \mathrm{~nm}$, continuous wave, $\left.23 \mathrm{~J} / \mathrm{cm}^{2}\right)$, but the effect did not reach statistical significance $(\mathrm{p}>0.05)$ [52]. In contrast, another rodent study found a negative effect of LLLT, indicating that incisors which underwent LLLT (GaAlAs, $780 \mathrm{~nm}$, continuous wave, $20 \mathrm{~J} / \mathrm{cm}^{2}$ ) exhibited higher rates of relapse compared with the control counterparts $(p<0.05)$ [53]. Interestingly, both rodent studies $[52,53]$ found that the number of osteoblasts was increased surrounding the laser-irradiated teeth, and one of them found an increase in the expression of matrix metalloproteinases in the LLLT group [53].

\section{Effects on orthodontic-induced inflammatory root resorption (OIIRR)}

To date, the number of studies investigating the effects of LLLT on OIIRR after OTM is limited, but the existing evidence is relatively conclusive and of high quality. One RCT [50] and two animal experiments $[54,55]$ showed some level of rehabilitative effect of LLLT on root surfaces. The detailed information of all included studies is summarised in Table 2. 


\begin{tabular}{|c|c|c|c|c|c|c|c|c|c|c|}
\hline \multirow{2}{*}{$\begin{array}{l}\text { Study } \\
\text { type }\end{array}$} & \multirow{2}{*}{$\begin{array}{l}\text { Authors } \\
\text { and } \\
\text { year }\end{array}$} & \multirow{2}{*}{$\begin{array}{l}\text { Study } \\
\text { model/ } \\
\text { design }\end{array}$} & \multirow{2}{*}{$\begin{array}{l}\text { Participants } \\
\text { and teeth }\end{array}$} & \multicolumn{3}{|l|}{ Interventions } & \multicolumn{3}{|l|}{ Assessment } & \multirow[t]{2}{*}{ Compi } \\
\hline & & & & $\begin{array}{l}\text { Active } \\
\text { treatment } \\
\text { strategy and } \\
\text { period }\end{array}$ & $\begin{array}{l}\text { Post-OTM } \\
\text { strategy }\end{array}$ & $\begin{array}{l}\text { Observation } \\
\text { time }\end{array}$ & Measure method & $\begin{array}{l}\text { Targeted } \\
\text { regions }\end{array}$ & $\begin{array}{l}\text { Outcome } \\
\text { variables }\end{array}$ & \\
\hline $\begin{array}{l}\text { Clinical } \\
\text { study }\end{array}$ & $\begin{array}{l}\text { Khaw } \\
\text { et al } \\
(2017) \\
{[50]}\end{array}$ & $\begin{array}{l}\text { RCT, } \\
\text { SMD }\end{array}$ & $\begin{array}{l}\mathrm{N}=20, \mathrm{n}=40 \\
(\mathrm{Nm}=12, \\
\mathrm{Nf}=8) \text { aged } \\
13-19 \text { years }\end{array}$ & $\begin{array}{l}\text { Buccal } \\
\text { cantilever } \\
\text { (Self-ligating } \\
\text { SPEED } \\
\text { brackets and } \\
\text { beta III } \\
\text { titanium, } \\
150 \mathrm{~g} \text { buccal } \\
\text { tipping } \\
\text { force); } 4 \mathrm{w}\end{array}$ & $\begin{array}{l}0.018 \text { SS } \\
\text { FA }\end{array}$ & $\nabla 6 w$ & micro-CT & $\begin{array}{l}\text { Extracted } \\
\text { first } \\
\text { premolars }\end{array}$ & $\begin{array}{l}\triangle \text { crater } \\
\text { volume }\end{array}$ & 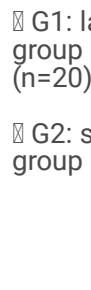 \\
\hline
\end{tabular}

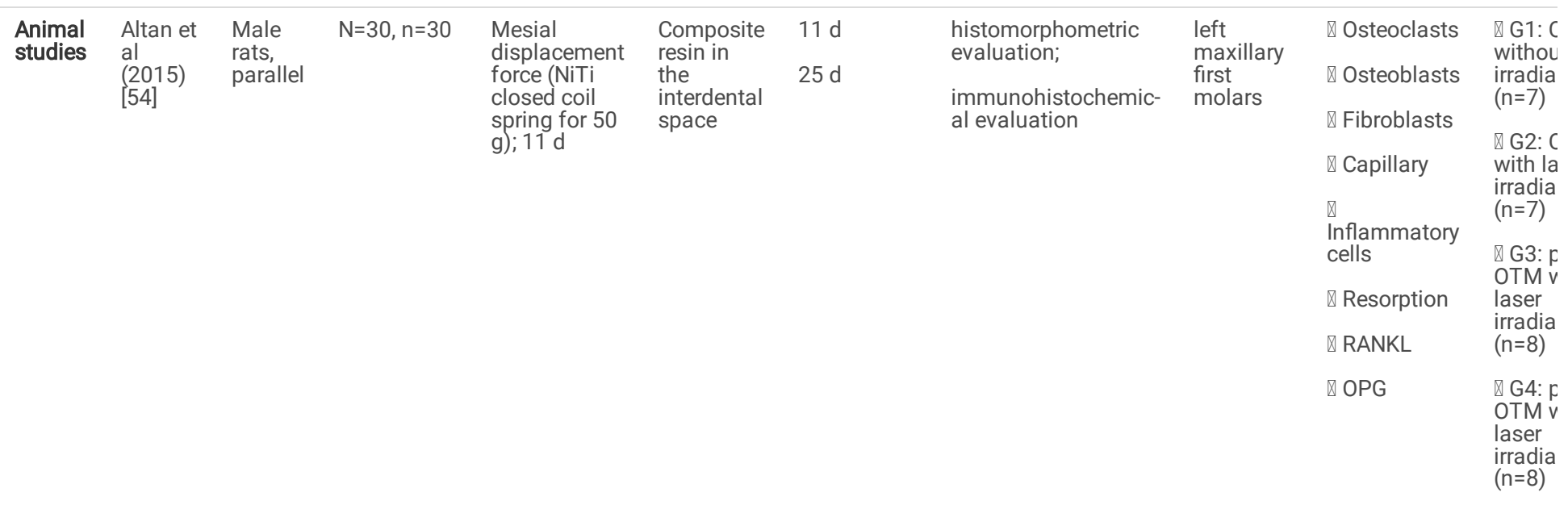

\begin{tabular}{|c|c|c|c|c|c|c|c|c|c|}
\hline \multirow{3}{*}{$\begin{array}{l}\text { de } \\
\text { Melo } \\
\text { Conti et } \\
\text { al. } \\
(2019) \\
{[55]}\end{array}$} & \multirow[t]{3}{*}{$\begin{array}{l}\text { Male } \\
\text { rats, } \\
\text { SMD }\end{array}$} & \multirow[t]{3}{*}{$\mathrm{N}=20, \mathrm{n}=40$} & \multirow{3}{*}{$\begin{array}{l}\text { Mesial } \\
\text { displacement } \\
\text { force (NiTi } \\
\text { closed coil } \\
\text { spsring for } \\
50 \mathrm{~g}) ; 7 \mathrm{~d}\end{array}$} & \multirow[t]{3}{*}{$\begin{array}{l}\text { No } \\
\text { retention }\end{array}$} & \multirow[t]{3}{*}{$\begin{array}{l}7 d \\
14 d\end{array}$} & \multirow{3}{*}{$\begin{array}{l}\text { histomorphometric } \\
\text { evaluation; } \\
\text { immunohistochemic- } \\
\text { al evaluation }\end{array}$} & \multirow{3}{*}{$\begin{array}{l}\text { first } \\
\text { maxillary } \\
\text { molars }\end{array}$} & \multirow{2}{*}{$\begin{array}{l}\triangle \text { Root } \\
\text { resorption } \\
\text { (RR) } \\
\text { Q RANKL }\end{array}$} & \multirow{2}{*}{$\begin{array}{l}\triangle \mathrm{G} 1: n \\
\text { OTM }( \\
\square \mathrm{G} 2: \mathrm{C} \\
(\mathrm{n}=10)\end{array}$} \\
\hline & & & & & & & & & \\
\hline & & & & & & & & $\triangle O P G$ & $\begin{array}{l}\text { Q G3: C } \\
\text { 7-day } \\
\text { healin! } \\
\text { withou } \\
(n=10)\end{array}$ \\
\hline
\end{tabular}


CCT: clinical controlled trial; SMD: split-mouth design; N: number of participants; n: number of evaluated teeth; FA: fixed appliances; CSF: circumferential sup MMP: matrix metalloproteinase; LLLT: low-level laser therapy

Table 2

Characteristics of included studies on orthodontically-induced inflammatory root resorption

\section{Clinical studies}

The one RCT that prescribed LLLT (AIGalnP, $660 \mathrm{~nm}$, continuous wave, $3.6 \mathrm{~J} / \mathrm{cm}^{2}$ ) applied it to one side of patients' upper first premolars immediately after the removal of a buccal tipping force during retention, whereas their counterparts on the opposite side were subject to a placebo laser. Six weeks post OTM, the mean total crater volume on the root surfaces of laser-irradiated teeth was $0.033 \pm 0.039 \mathrm{~mm}^{3}$ less than that of the placebo-irradiated teeth. However, this difference did not exhibit statistical significance $(p>0.05)[50]$.

\section{Animal studies}

Of the two animal studies both adopted rodent models, one used a parallel study design involving 30 subjects and 30 maxillary first molars for the outcome assessment [54], whereas the other study applied a split-mouth design including 20 subjects with 40 paired teeth in total [55]. They both found that teeth underwent GaAlAs laser irradiation ( $820 \mathrm{~nm}$, continuous wave, $4.8 \mathrm{~J} / \mathrm{cm}^{2}$ [54], and $810 \mathrm{~nm}$, continuous wave, $75 \mathrm{~J} / \mathrm{cm}^{2}$ [55]) showed less OIIRR compared with their control counterparts $(\mathrm{p}<0.05)$. In addition, both studies found that the number of clastic cells and the ratio of receptor activator of nuclear factor kappa-B ligand to osteoprotegerin (RANKL/OPG) decreased significantly in LLLT-irradiated teeth compared with their control counterparts, indicating that this positive impact was associated with the downregulation of clastogenic activities [54,55]. Moreover, one study determined that the numbers of osteoblasts and fibroblasts surrounding the teeth in the laser-irradiated group were also remarkably increased, especially when LLLT was delivered after the active orthodontic treatment compared with irradiation during OTM [54]. This suggests that LLLT is more likely to have a restorative effect than a preventive effect on root resorption.

Although the results of the animal studies were in line with the evidence provided by the RCT, variations between all included studies were noted, such as the laser types (AlGalnP [50] or GaAlAs [54, 55]), force durations (7 [55], 11 [54] or 28 [50] days), laser parameters (660 nm, $75 \mathrm{~mW}$ [50]; $820 \mathrm{~nm}, 50 \mathrm{~mW}$ [54]; or $810 \mathrm{~nm}, 100 \mathrm{~mW}$ [55]), and retention regimens (6-week retention [50], 2-week retention [54]; or no retainer [55]). Whether these factors affect the magnitude of LLLT's effects on OIIRR was not discussed. The summary of LLLT regimens adopted by all included studies is presented in Table 3. 


\begin{tabular}{|c|c|c|c|c|c|c|c|c|c|c|c|c|}
\hline $\begin{array}{l}\text { Study } \\
\text { types }\end{array}$ & Outcome & $\begin{array}{l}\text { Authors } \\
\text { and year }\end{array}$ & $\begin{array}{l}\text { Laser } \\
\text { type }\end{array}$ & $\begin{array}{l}\text { Wavelength } \\
(\mathrm{nm})\end{array}$ & $\begin{array}{l}\text { Beam } \\
\text { size } \\
\left(\mathrm{cm}^{2}\right)\end{array}$ & $\begin{array}{l}\text { Output } \\
\text { power } \\
\text { (mW) }\end{array}$ & Mode & $\begin{array}{l}\text { Dosage } \\
\text { density } \\
\left(\mathrm{J} / \mathrm{cm}^{2}\right)\end{array}$ & $\begin{array}{l}\text { Dosage } \\
\text { (J) }\end{array}$ & $\begin{array}{l}\text { Timing for } \\
\text { first } \\
\text { irradiation }\end{array}$ & $\begin{array}{l}\text { Frequency } \\
\text { and times }\end{array}$ & $\begin{array}{l}\text { Irradiation } \\
\text { period per } \\
\text { session } \\
\text { (s) }\end{array}$ \\
\hline \multirow[t]{3}{*}{$\begin{array}{l}\text { Clinical } \\
\text { studies }\end{array}$} & OR & $\begin{array}{l}\text { Zahra et } \\
\text { al (2009) } \\
\text { [48] }\end{array}$ & GaAs & 904 & 0.5 & 30 & pw & 4.9 & 16.2 & $\begin{array}{l}\text { within the } \\
\text { first week } \\
\text { after } \\
\text { diastema } \\
\text { closed but } \\
\text { before } \\
\text { debonding }\end{array}$ & $\begin{array}{l}\text { every } \\
\text { second } \\
\text { day (3 } \\
\text { sessions) }\end{array}$ & 180 \\
\hline & & $\begin{array}{l}\text { Jahanbin } \\
\text { et al } \\
(2014) \\
{[49]}\end{array}$ & GaAlAs & 810 & 0.28 & 200 & $\mathrm{cW}$ & 35.7 & 80 & $\begin{array}{l}\text { at the } \\
\text { finishing } \\
\text { stage of } \\
\text { orthodontic } \\
\text { treatment }\end{array}$ & $\begin{array}{l}\text { twice a } \\
\text { week (8 } \\
\text { sessions) }\end{array}$ & 200 \\
\hline & OIIRR & $\begin{array}{l}\text { Khaw et } \\
\text { al }(2017) \\
{[50]}\end{array}$ & AlGalnP & 660 & 0.26 & 75 & $\mathrm{cW}$ & 3.6 & 45 & $\begin{array}{l}4 \text { weeks } \\
\text { after active } \\
\text { orthodontic } \\
\text { force }\end{array}$ & $\begin{array}{l}\text { Once a } \\
\text { week (6 } \\
\text { sessions) }\end{array}$ & 120 \\
\hline \multirow[t]{4}{*}{$\begin{array}{l}\text { Animal } \\
\text { studies }\end{array}$} & OR & $\begin{array}{l}\text { Kim et al } \\
(2010) \\
{[51]}\end{array}$ & GaAlAs & 808 & NR & 763 & pw & $\begin{array}{l}4.63- \\
6.47\end{array}$ & NR & $\begin{array}{l}\text { Immediately } \\
\text { after } \\
\text { orthodontic } \\
\text { couple force } \\
\text { removed }\end{array}$ & $\begin{array}{l}\text { every } 3 \\
\text { days (9 } \\
\text { sessions) }\end{array}$ & 240 \\
\hline & & $\begin{array}{l}\text { Franzen } \\
\text { et al } \\
(2015) \\
{[52]}\end{array}$ & GaAlAs & 830 & 0.13 & 75 & $\mathrm{cW}$ & 23 & $3-21$ & $\begin{array}{l}\text { Immediately } \\
\text { after } \\
\text { appliance } \\
\text { removal }\end{array}$ & $\begin{array}{l}\text { Evenly } \\
\text { distributed } \\
\text { during } \\
\text { relapse } \\
\text { period }(1, \\
2,3,4,5,7 \\
\text { sessions) }\end{array}$ & 17 \\
\hline & & $\begin{array}{l}\text { Lee et al } \\
(2016) \\
{[53]}\end{array}$ & GaAlAs & 780 & NR & NR & $\mathrm{cw}$ & 20 & NR & $\begin{array}{l}\text { after } 1 \text {-week } \\
\text { temporal } \\
\text { retention }\end{array}$ & $\begin{array}{l}\text { Daily until } \\
\text { sacrificed } \\
\text { ( } 2,4,6 \\
\text { sessions) }\end{array}$ & NR \\
\hline & OIIRR & Altan et & GaAlAs & 820 & 0.208 & 50 & $\mathrm{cW}$ & 4.8 & 4.2 & After 11-day & Every & 12 \\
\hline
\end{tabular}


OTM and removal of force appliance other day

for 2 weeks (7 sessions)

\begin{tabular}{|c|c|c|c|c|c|c|c|c|c|}
\hline $\begin{array}{l}\text { de Melo } \\
\text { Conti et } \\
\text { al (2019) } \\
{[55]}\end{array}$ & GaAlAs & 810 & 0.02 & 100 & $\mathrm{cW}$ & 75 & 12 & $\begin{array}{l}\text { After 7-day } \\
\text { OTM and } \\
\text { removal of } \\
\text { force } \\
\text { apparatus }\end{array}$ & $\begin{array}{l}\text { Day } 7,9 \\
11, \text { and } 13 \\
\text { (4 } \\
\text { sessions) }\end{array}$ \\
\hline
\end{tabular}

OR: orthodontic relapse; OIIRR: orthodontically-induced inflammatory root resorption; GaAs: gallium-arsenide; GaAlAs: gallium-aluminum-arsenide; AlGalnP: á gallium-indium-phosphide; cw: continuous wave; pw: pulse wave; NR: not reported

Table 3

Detailed low-level laser regimens for all included studies

\section{Quality evaluation}

\section{Clinical studies}

The quality assessment of the three human studies was performed according to the guidelines of the Cochrane Risk of Bias Tool. The two CCTs [48, 49] were ranked as having a high risk of bias with emphasise on the lack of blinding and randomisation. Additionally, they conducted no power analysis or sample size calculation, which further diminished the quality of evidence. In comparison, the risk of bias of the RCT for the effects of LLLT on OIIRR ranked considerably lower, as all seven evaluation domains were fulfilled [50] (Fig. 2)

\section{Animal studies}

The qualities of the animal studies were assessed using the SYRCLE tool. All animal studies were ranked as possessing a high risk of bias due to their lack of fulfilment of or unclear declaration of randomisation, balancing subjects' characteristics, blinding during intervention and assessment, or avoidance of data attrition [51-55]. The schematic representation of the assessment results of risk of bias in animal studies is provided in Fig. 3.

\section{Discussion}

Of all the included studies evaluating the effects of LLLT on post-OTM tooth stability, two discussed rotational relapse. In clinical practice, orthodontically derotated teeth are more likely to return to their original state, even when orthodontic retainers are routinely administered. Past studies have revealed that soft tissue turnover, i.e. the remodelling of collagen and elastic fibres, plays a vital role in the occurrence of rotational relapse [56]. Based on this finding, some researchers hypothesised that the biomodulation of soft tissues by LLLT might be a promising approach to prevent post-OTM rotation. However, according to the results of the two relevant studies, the impacts of LLLT could be either positive or negative depending on various factors to the maintenance of orthodontically de-rotated tooth position. In line with the biphasic dosage-response theory [57], the first factor is the dosage density. Jahanbin et al. [49] found that a GaAlAs laser with 810-nm wavelength could alleviate the degree of rotational relapse when the dosage density was high at $35.7 \mathrm{~J} / \mathrm{cm}^{2}$. In contrast, Kim et al. [51] used the same type of laser (GaAlAs, $808 \mathrm{~nm}$ ) with a low dosage density $\left(4.63-6.47 \mathrm{~J} / \mathrm{cm}^{2}\right.$ ) and found that it decreased post-treatment tooth stability. One systematic review of past in vitro studies reported that laser with a dosage density of less than $16 \mathrm{~J} / \mathrm{cm}^{2} \mathrm{could}$ promote fibroblast growth, proliferation and osteogenic differentiation, where laser with an extremely high dosage density exhibited inhibitory effects [25]. It is possible that the effects of LLLT on rotational relapse also follow the same rules, converting fibroblasts from predominantly anabolic activities to catabolic ones corresponding to a shift from adverse effects to positive ones along with the increase in dosage density [42]. However, this interpretation only applies to teeth free of movement after the immediate termination of active forces. As Kim et al [58] suggests, LLLT could act differently to the orthodontic outcomes for teeth prescribed with retainers and those without by stimulating soft tissue metabolism. Therefore, whether the adjunctive LLLT has extra benefits on the efficacy of conventional retention appliances is still unclear. Finally, there are other confounders that hinder any generalisation of the effects of LLLT on rotational relapse, including the substantial heterogeneities in the characteristics of subjects and the initial status of the experimental teeth. In addition, both articles have a high risk of bias because of a limited number of recruited subjects and no sample size calculations. Further investigations with a higher quality of evidence are thus highly warranted.

The other two post-OTM outcomes discussed by the remaining six studies, i.e. transitional relapse and root resorption, are both closely related to the activities of osteoblast-like cells and osteoclast-like cells for hard tissue remodelling. On one hand, after the termination of active forces, alveolar processes generate some hyalinised areas in response to the released mechanical forces, which then trigger osteoclast recruitment and bone resorption in the direction of tooth 
relapse on the previous tension side. Meanwhile, more anabolic activities such as osteoblast proliferation and differentiation occur on the opposite side, leading to bone regeneration against the direction of tooth relapse to compensate for previous bone resorption $[16,17,60]$. On the other hand, pathological OIIRR occur during OTM when osteoclastic-like cells accumulate near the root surfaces [61, 62]. After orthodontic force removal, a physiological repair would follow involving the deposition of uncalcified-cementoid matrix, fibroblast-like cells and cementoblast cells as well as the detachment of clastic cells [63]. Past cellular [28] and molecular investigations [64] have documented LLLT's capacity to modulate the activities of osteoblasts and osteoclasts with bone-related biomarkers such as RANKL and OPG. This provides a biological explanation for adopting LLLT to prevent transitional relapse and OIIRR. However, this theory is yet to be supported.

Among the three studies on transitional relapse [48,52,53], two failed to observe any significant reduction in the amount of post-OTM displacement for LLLTirradiated teeth after the termination of active forces [48, 52]. One study showed a statistically significant detrimental effect of LLLT on tooth position maintenance [53]. This inconsistency in the results on transitional relapse might also be related to the diversity of laser types and parameter settings, similar to the results on rotational relapse. However, one past review [28] reported a general susceptibility of osteoblast-like cells to multiple laser parameters without a clear specificity, unless the dosage densities are extremely high. Thus, the disagreement between the three studies is possibly dominated by the variation in their retention regimens. Two studies implemented LLLT immediately after OTM [48, 52], whereas the other one delivered irradiation after a 1 -week retention [53]. Because the lag of adjacent alveolar reconstruction is the primary reason for transitional relapse, the effects of LLLT on tooth position maintenance might omit the critical period and recede with time.

Comparably, the results of the three studies on OIIRR are more consistent [50,54,55]. The two animal studies reported favourable effects of LLLT with statistically significant reductions in OIIRR for teeth that received LLLT during the post-OTM period compared with their counterparts [54, 55], and one clinical trial showed a general tendency for a positive effect of LLLT but without a statistical significance [50]. These beneficial effects of LLLT in the reduction of OIIRR are in line with previous findings $[65,66]$ suggesting that LLLT could enhance the development of roots or their reparative process by stimulating the proliferation of cementoblast cells and the formation of secondary cementum. The two rodent studies applied near-infrared light at 810 and $820 \mathrm{~nm}$ [54, 55], whereas the clinical study adopted an AIGalnP laser at a 660-nm wavelength [50]. Considering the penetration depth of LLLT's PBM is largely dependent on the wavelength [67] and that the longer is the wavelength the deeper can photons travel through biological surfaces [68], it might be possible that the nonsignificant differences found in the clinical study were because the PBM effect cannot occur at the depth of root surfaces. The second reason might be the frequency of repeated laser applications, as longer time intervals and total irradiation spans in the clinical study might vary the effects on OIIRR [50]. Finally, the substantial differences in the study subjects and retention regimens might also blur the interpretation of the above inconsistencies and whether the effects reached statistical significance.

It is clear that current evidence is still insufficient to deduce the effects of LLLT on the prognosis of orthodontic treatment after the active OTM stage, in terms of outcomes for both tooth relapse and OIIRR. One hinderance in generalising these results is the aforementioned methodological discrepancies, and another is the fact that the underlying cellular and molecular mechanisms are yet to be fully clarified. A classical theory for the PBM effect considers the activities of cytochrome $\mathrm{C}$ oxidase (CCO) in the respiratory chain, which are boosted by photons in the red and infrared wavelengths that penetrate the mitochondria [69]. By inducing high levels of adenosine triphosphate (ATP) production and vital second messengers such as nitric oxide and reactive oxygen species, LLLT regulates various metabolic activities such as cell proliferation, migration, adhesion and apoptosis. However, this theory cannot explain the inconsistencies between some therapeutic laser wavelengths and the absorption spectra of CCO. Another hypothesis, the 'water oscillator paradox', was proposed by SantanaBlank et al. [70] implicating intracellular water dynamics also play an essential role in PBM effects. Recently, Wang et al. [71] found that heat/light-gated ion channels seem to be the primary photoreceptor for $980-\mathrm{nm}$ wavelength lasers, whereas CCO is the primary photoreceptor for the 810-nm wavelength. Most studies reviewed here used LLLT in the 808-830-nm wavelength [49,51,52,54, 55], except for one study that used a 660-nm laser for OIIRR [50] and two that used 780-nm [53] and 904-nm [48] lasers for transitional relapse. Considering that chromophores might alternate with different wavelengths, the optimal dosage for achieving favourable PBM effects can vary and may significantly influence post-OTM outcomes.

This systematic review is the first to comprehensively evaluate the effects of LLLT in post-OTM scenarios, aiming to justify the application of LLLT for plausible orthodontic prognosis. According to our results, there is great controversy over the effects of LLLT on orthodontic relapse, but its use in post-OTM applications for reparative effects on root resorption are generally recommended. However, great heterogeneity was noted in the assessed outcomes, study subjects, OTM and post-OTM regimens and LLLT parameters. Moreover, most studies suffered from limitations including small sample sizes, high risk of bias, relatively short observation periods and a shortage in demonstrations of cellular and molecular changes. Therefore, more well-designed studies with broader LLLT parameters and more consistent orthodontic and post-OTM settings are highly anticipated in the near future.

\section{Declarations}

\section{Ethical approval and consent to participate}

The protocol has been prospectively registered on the PROSPERO online database (CRD49019132133).

\section{Consent for publication}

Not applicable

\section{Availability of data and materials}


All data generated or analysed during this study are included in this published article and its supplementary information files.

\section{Competing interest}

The authors declare that they have no competing interests.

\section{Funding}

The authors received no financial support for the research, authorship, and publication of this article.

\section{Authors' contributions}

ZYS and KWFW performed the systematic search, risk of bias assessment and data synthesis, ZYS drafted the manuscript, CMG and GM reviewed the paper and made constructive revisions, and YQY is responsible for communicating with the other authors about progress, submissions of revisions and final approval of proofs. We confirm that the manuscript has been read and approved by all named authors and that there are no other persons who satisfied the criteria for authorship but are not listed. We further confirm that the order of authors listed in the manuscript has been approved by all of us.

\section{Acknowledgements}

Not applicable

\section{List Of Abbreviations}

\begin{tabular}{|ll|}
\hline LLLT & low-level laser therapy \\
\hline OTM & orthodontic tooth movement \\
\hline OIIRR & orthodontically-induced inflammatory root resorption \\
\hline PBM & photobiomodulation \\
\hline RCT & randomized-controlled trial \\
\hline CCT & controlled-clinical trial \\
CCO & cytochrome C oxidase \\
\hline ATP & adenosine triphosphate \\
\hline RANKL/OPG & receptor activator of nuclear factor kappa-B ligand to osteoprotegerin \\
\hline SMD & split-mouth design \\
\hline FA & fixed appliance \\
\hline CSF & circumferential supracrestal fiberotomy \\
\hline MMP & matrix metalloproteinase \\
\hline GaAs & gallium-arsenide \\
\hline GaAIAs & gallium-aluminum-arsenide \\
\hline AIGalnP & aluminum- gallium-indium-phosphide \\
\hline CW/pw & continuous wave / pulse wave \\
\hline
\end{tabular}

\section{References}

1. Steinnes J, Johnsen G, Kerosuo H. Stability of orthodontic treatment outcome in relation to retention status: An 8-year follow-up. American Journal of Orthodontics and Dentofacial Orthopedics. 2017 Jun 1;151(6):1027-33.

2. Littlewood SJ, Millett DD, Doubleday BB, Bearn DD. Retention procedures for stabilising tooth position after treatment with orthodontic braces. Cochrane Database of Systematic Reviews. 2000(2).

3. Edwards JG. A long-term prospective evaluation of the circumferential supracrestal fiberotomy in alleviating orthodontic relapse. American Journal of Orthodontics and Dentofacial Orthopedics. 1988 May 1;93(5):380-7.

4. Hirate $\mathrm{Y}$, Yamaguchi M, Kasai K. Effects of relaxin on relapse and periodontal tissue remodeling after experimental tooth movement in rats. Connective tissue research. 2012 Jun 1;53(3):207-19. 
5. Hassan AH, Al-Hubail A, Al-Fraidi AA. Bone inductive proteins to enhance postorthodontic stability: A pilot study. The Angle Orthodontist. 2010 Nov;80(6):1051-60.

6. Alhasyimi AA, Pudyani PP, Asmara W, Ana ID. Enhancement of post-orthodontic tooth stability by carbonated hydroxyapatite-incorporated advanced platelet-rich fibrin in rabbits. Orthodontics \& craniofacial research. 2018 May;21(2):112-8.

7. Dolci GS, Portela LV, de Souza DO, Fossati AC. Atorvastatin-induced osteoclast inhibition reduces orthodontic relapse. American Journal of Orthodontics and Dentofacial Orthopedics. 2017 Mar 1;151(3):528-38.

8. Liu Y, Zhang T, Zhang C, Jin SS, Yang RL, Wang XD, Jiang N, Gan YH, Kou XX, Zhou YH. Aspirin blocks orthodontic relapse via inhibition of CD4+ T lymphocytes. Journal of dental research. 2017 May;96(5):586-94.

9. Atsawasuwan P, Shirazi S. Advances in Orthodontic Tooth Movement: Gene Therapy and Molecular Biology Aspect. InCurrent Approaches in Orthodontics 2018 Nov 5. IntechOpen.

10. Reitan K. Tissue rearrangement during retention of orthodontically rotated teeth. The Angle Orthodontist. 1959 Apr;29(2):105-13.

11. Boese LR. Increased stability of orthodontically rotated teeth following gingivectomy in Macaca nemestrina. American Journal of Orthodontics and Dentofacial Orthopedics. 1969 Sep 1;56(3):273-90.

12. Edwards JG. A surgical procedure to eliminate rotational relapse. American Journal of Orthodontics and Dentofacial Orthopedics. 1970 Jan 1;57(1):35-46.

13. Redlich M, Rahamim E, Gaft A, Shoshan S. The response of supraalveolar gingival collagen to orthodontic rotation movement in dogs. American journal of orthodontics and dentofacial orthopedics. 1996 Sep 1;110(3):247-55.

14. Meng M, Lv C, Yang Q, He S, Wu S, Liu Y, Zou J, Zhou X, Chen S. Expression of proteins of elastic fibers and collagen type I in orthodontically rotated teeth in rats. American Journal of Orthodontics and Dentofacial Orthopedics. 2018 Aug 1;154(2):249-59.

15. Henneman S, Reijers RR, Maltha JC, Von den Hoff JW. Local variations in turnover of periodontal collagen fibers in rats. Journal of periodontal research. 2012 Jun;47(3):383-8.

16. Franzen TJ, Brudvik P, Vandevska-Radunovic V. Periodontal tissue reaction during orthodontic relapse in rat molars. The European Journal of Orthodontics. 2013 Apr 1;35(2):152-9.

17. Franzen TJ, Monjo M, Rubert M, Vandevska-Radunovic V. Expression of bone markers and micro-CT analysis of alveolar bone during orthodontic relapse. Orthodontics \& craniofacial research. 2014 Nov;17(4):249-58.

18. De Souza TO, Martins MA, Bussadori SK, Fernandes KP, Tanji EY, Mesquita-Ferrari RA, Martins MD. Clinical evaluation of low-level laser treatment for recurring aphthous stomatitis. Photomedicine and Laser Surgery. 2010 Oct 1;28(S2):S-85.

19. de Paula Eduardo C, de Freitas PM, Esteves-Oliveira M, Aranha AC, Ramalho KM, Simões A, Bello-Silva MS, Tunér J. Laser phototherapy in the treatment of periodontal disease. A review. Lasers in Medical Science. 2010 Nov 1;25(6):781-92.

20. Gojkov-Vukelic M, Hadzic S, Zukanovic A, Pasic E, Pavlic V. Application of diode laser in the treatment of dentine hypersensitivity. Medical Archives. 2016 Dec;70(6):466.

21. Ren C, McGrath C, Jin L, Zhang C, Yang Y. The effectiveness of low-level laser therapy as an adjunct to non-surgical periodontal treatment: a metaanalysis. Journal of periodontal research. $2017 \mathrm{Feb} ; 52(1): 8-20$.

22. Santos FT, Sciescia R, Santos PL, Weckwerth V, Pizzol KE, Queiroz TP. Is Low-Level Laser Therapy Effective on Sensorineural Recovery After Bilateral Sagittal Split Osteotomy? Randomized Trial. Journal of Oral and Maxillofacial Surgery. 2019 Jan 1;77(1):164-73.

23. Abergel RP, Lyons RF, Castel JC, Dwyer RM, Uitto J. Biostimulation of wound healing by lasers: experimental approaches in animal models and in fibroblast cultures. The Journal of dermatologic surgery and oncology. 1987 Feb;13(2):127-33.

24. Balboni GC, Brandi ML, Zonefrati L, Repice F. Effect of laser irradiation on collagen production by fibroblasts in vitro. Bulletin de l'Association des anatomistes. 1985 Mar;69(204):15-8.

25. Ren C, McGrath C, Jin L, Zhang C, Yang Y. Effect of diode low-level lasers on fibroblasts derived from human periodontal tissue: a systematic review of in vitro studies. Lasers in medical science. 2016 Sep 1;31(7):1493-510.

26. Kohale BR, Agrawal AA, Raut CP. Effect of low-level laser therapy on wound healing and patients' response after scalpel gingivectomy: A randomized clinical split-mouth study. Journal of Indian Society of Periodontology. 2018 Sep;22(5):419.

27. Amid R, Kadkhodazadeh M, Ahsaie MG, Hakakzadeh A. Effect of low level laser therapy on proliferation and differentiation of the cells contributing in bone regeneration. Journal of lasers in medical sciences. 2014;5(4):163.

28. Deana AM, de Souza AM, Teixeira VP, Mesquita-Ferrari RA, Bussadori SK, Fernandes KP. The impact of photobiomodulation on osteoblast-like cell: a review. Lasers in medical science. $2018 \mathrm{Jul}$ 1;33(5):1147-58.

29. Kiyosaki T, Mitsui N, Suzuki N, Shimizu N. Low-level laser therapy stimulates mineralization via increased Runx2 expression and ERK phosphorylation in osteoblasts. Photomedicine and laser surgery. 2010 Aug 1;28(S1):S-167.

30. Stein A, Benayahu D, Maltz L, Oron U. Low-level laser irradiation promotes proliferation and differentiation of human osteoblasts in vitro. Photomedicine and Laser Therapy. 2005 Apr 1;23(2):161-6.

31. Garcez AS, Suzuki SS, Martinez EF, lemini MG, Suzuki H. Effects of low-intensity laser therapy over mini-implants success rate in pigs. Lasers in medical science. 2015 Feb 1;30(2):727-32.

32. Ekizer A, Türker G, Uysal T, Güray E, Taşdemir Z. Light emitting diode mediated photobiomodulation therapy improves orthodontic tooth movement and miniscrew stability: A randomized controlled clinical trial. Lasers in surgery and Medicine. 2016 Dec;48(10):936-43. 
33. Yanaguizawa MS, Suzuki SS, Martinez EF, Suzuki H, Pelegrin MC, Garcez AS. Effects of low-level laser therapy in orthodontic patients on immediate inflammatory response after mini-implants insertion: a preliminary report. Photomedicine and laser surgery. 2017 Jan 1;35(1):57-63.

34. Skondra FG, Koletsi D, Eliades T, Farmakis ET. The effect of low-level laser therapy on bone healing after rapid maxillary expansion: a systematic review. Photomedicine and laser surgery. 2018 Feb 1;36(2):61-71.

35. Garcia VJ, Arnabat J, Comesaña R, Kasem K, Ustrell JM, Pasetto S, Segura OP, ManzanaresCéspedes MC, Carvalho-Lobato P. Effect of low-level laser therapy after rapid maxillary expansion: a clinical investigation. Lasers in medical science. 2016 Aug 1;31(6):1185-94.

36. Ferreira FN, Gondim JO, Neto JJ, dos Santos PC, de Freitas Pontes KM, Kurita LM, de Araújo MW. Effects of low-level laser therapy on bone regeneration of the midpalatal suture after rapid maxillary expansion. Lasers in medical science. $2016 \mathrm{Jul}$ 1;31(5):907-13.

37. Ren C, McGrath C, Gu M, Jin L, Zhang C, Sum FH, Wong KW, Chau AC, Yang Y. Low-level laser-aided orthodontic treatment of periodontally compromised patients: a randomised controlled trial. Lasers in Medical Science. 2020 Apr;35(3):729-39.

38. Domínguez A, Gómez C, Palma JC. Effects of low-level laser therapy on orthodontics: rate of tooth movement, pain, and release of RANKL and OPG in GCF. Lasers in medical science. 2015 Feb 1;30(2):915-23.

39. Doshi-Mehta G, Bhad-Patil WA. Efficacy of low-intensity laser therapy in reducing treatment time and orthodontic pain: a clinical investigation. American Journal of Orthodontics and Dentofacial Orthopedics. 2012 Mar 1;141(3):289-97.

40. Ren C, McGrath C, Yang Y. The effectiveness of low-level diode laser therapy on orthodontic pain management: a systematic review and meta-analysis. Lasers in medical science. 2015 Sep 1;30(7):1881-93.

41. Sonesson M, De Geer E, Subraian J, Petrén S. Efficacy of low-level laser therapy in accelerating tooth movement, preventing relapse and managing acute pain during orthodontic treatment in humans: a systematic review. BMC Oral Health. 2017 Dec;17(1):11.

42. Meng M, Yang M, Lv C, Yang Q, Yang Z, Chen S. Effect of low-level laser therapy on relapse of rotated teeth: a systematic review of human and animal study. Photomedicine and laser surgery. 2017 Jan 1;35(1):3-11.

43. Moher D, Liberati A, Tetzlaff J, Altman DG. Preferred reporting items for systematic reviews and meta-analyses: the PRISMA statement. Annals of internal medicine. 2009 Aug 18;151(4):264-9.

44. Liberati A, Altman DG, Tetzlaff J, Mulrow C, Gøtzsche PC, loannidis JP, Clarke M, Devereaux PJ, Kleijnen J, Moher D. The PRISMA statement for reporting systematic reviews and meta-analyses of studies that evaluate health care interventions: explanation and elaboration. Annals of internal medicine. 2009 Aug 18;151(4):W-65.

45. Collaboration C. Review Manager (RevMan)[Computer Program] Version 5.2. 3, The Nordic Cochrane Centre, Copenhagen, 2012. HEALTH PSYCHOLOGY REVIEW. 2014;17.

46. Cochrane Handbook for Systematic Reviews of Interventions. Available at: http://handbook.cochrane.org/chapter_ 8/8_5_the_cochrane_collaborations_tool_for_assessing_risk_of_bias.htm (Last accessed August 4, 2016).

47. Hooijmans CR, Rovers MM, De Vries RB, Leenaars M, Ritskes-Hoitinga M, Langendam MW. SYRCLE's risk of bias tool for animal studies. BMC medical research methodology. 2014 Dec 1;14(1):43.

48. Zahra SE, Elkasi AA, Eldin MS, Vandevska-Radunovic V. The effect of low level laser therapy (LLLT) on bone remodelling after median diastema closure: a one year and half follow-up study. orthodontic waves. 2009 Sep 1;68(3):116-22.

49. Jahanbin A, Ramazanzadeh B, Ahrari F, Forouzanfar A, Beidokhti M. Effectiveness of Er: YAG laser-aided fiberotomy and low-level laser therapy in alleviating relapse of rotated incisors. American Journal of Orthodontics and Dentofacial Orthopedics. 2014 Nov 1;146(5):565-72.

50. Khaw CM, Dalci O, Foley M, Petocz P, Darendeliler MA, Papadopoulou AK. Physical properties of root cementum: Part 27. Effect of low-level laser therapy on the repair of orthodontically induced inflammatory root resorption: A double-blind, split-mouth, randomized controlled clinical trial. American Journal of Orthodontics and Dentofacial Orthopedics. 2018 Sep 1;154(3):326-36.

51. Kim SJ, Paek JH, Park KH, Kang SG, Park YG. Laser-aided circumferential supracrestal fiberotomy and low-level laser therapy effects on relapse of rotated teeth in beagles. The Angle orthodontist. 2010 Mar;80(2):385-90.

52. Franzen TJ, Zahra SE, El-Kadi A, Vandevska-Radunovic V. The influence of low-level laser on orthodontic relapse in rats. European journal of orthodontics. 2015 Feb 1;37(1):111-7.

53. Lee SH, Kim KA, Anderson S, Kang YG, Kim SJ. Combined effect of photobiomodulation with a matrix metalloproteinase inhibitor on the rate of relapse in rats. The Angle Orthodontist. 2016 Mar;86(2):206-13.

54. Altan AB, Bicakci AA, Mutaf HI, Ozkut M, Inan VS. The effects of low-level laser therapy on orthodontically induced root resorption. Lasers in medical science. 2015 Nov 1;30(8):2067-76.

55. de Melo Conti C, Suzuki H, Garcez AS, Suzuki SS. Effects of photobiomodulation on root resorption induced by orthodontic tooth movement and RANKL/OPG expression in rats. Photochemistry and photobiology. 2019 Sep;95(5):1249-57.

56. Bumann A, Carvalho RS, Schwarzer CL, Yen EH. Collagen synthesis from human PDL cells following orthodontic tooth movement. European journal of orthodontics. 1997 Feb 1;19(1):29-37.

57. Huang YY, Chen AC, Carroll JD, Hamblin MR. Biphasic dose response in low level light therapy. Dose-response. 2009 Oct 1;7(4):dose-response.

58. Kim SJ, Kang YG, Park JH, Kim EC, Park YG. Effects of low-intensity laser therapy on periodontal tissue remodeling during relapse and retention of orthodontically moved teeth. Lasers in medical science. 2013 Jan 1;28(1):325-33.

59. Lovatt R, Goonewardene M, Tennant M. Relapse following orthodontic rotation of teeth in dogs. Australian orthodontic journal. 2008 May;24(1):5.

60. Yoshida Y, Sasaki T, Yokoya K, Hiraide T, Shibasaki Y. Cellular roles in relapse processes of experimentally-moved rat molars. Microscopy. 1999 Jan;48(2):147-57.

Page 14/16 
61. Brudvik P, Rygh P. Transition and determinants of orthodontic root resorption-repair sequence. The European Journal of Orthodontics. 1995 Jun 1;17(3):177-88.

62. Brudvik P, Rygh P. The repair of orthodontic root resorption: an ultrastructural study. The European Journal of Orthodontics. 1995 Jun 1;17(3):189-98.

63. Currell SD, Liaw A, Grant PD, Esterman A, Nimmo A. Orthodontic mechanotherapies and their influence on external root resorption: a systematic review. American Journal of Orthodontics and Dentofacial Orthopedics. 2019 Mar 1;155(3):313-29.

64. Hosseinpour S, Fekrazad R, Arany PR, Ye Q. Molecular impacts of photobiomodulation on bone regeneration: a systematic review. Progress in biophysics and molecular biology. 2019 Apr 17.

65. Toomarian L, Fekrazad R, Tadayon N, Ramezani J, Tunér J. Stimulatory effect of low-level laser therapy on root development of rat molars: a preliminary study. Lasers in medical science. 2012 May 1;27(3):537-42.

66. Alsulaimani M, Doschak M, Dederich D, Flores-Mir C. Effect of low-level laser therapy on dental root cementum remodeling in rats. Orthodontics \& craniofacial research. 2015 May;18(2):109-16.

67. Barolet D. Light-emitting diodes (LEDs) in dermatology. Seminars in cutaneous medicine and surgery 2008 Dec 31 (Vol. 27, No. 4, pp. 227-238). No longer published by Elsevier.

68. Avci P, Gupta A, Sadasivam M, Vecchio D, Pam Z, Pam N, Hamblin MR. Low-level laser (light) therapy (LLLT) in skin: stimulating, healing, restoring. InSeminars in cutaneous medicine and surgery 2013 Mar (Vol. 32, No. 1, p. 41). NIH Public Access.

69. Wong-Riley MT, Liang HL, Eells JT, Chance B, Henry MM, Buchmann E, Kane M, Whelan HT. Photobiomodulation directly benefits primary neurons functionally inactivated by toxins role of cytochrome c oxidase. Journal of Biological Chemistry. 2005 Feb 11;280(6):4761-71.

70. Santana-Blank L, Rodriguez-Santana E, Santana-Rodriguez K. Theoretic, experimental, clinical bases of the water oscillator hypothesis in near-infrared photobiomodulation. Photomedicine and laser surgery. 2010 Aug 1;28(S1):S-41.

71. Wang Y, Huang YY, Wang Y, Lyu P, Hamblin MR. Photobiomodulation of human adipose-derived stem cells using $810 \mathrm{~nm}$ and $980 \mathrm{~nm}$ lasers operates via different mechanisms of action. Biochimica et Biophysica Acta (BBA)-General Subjects. 2017 Feb 1;1861(2):441-9.

\section{Figures}

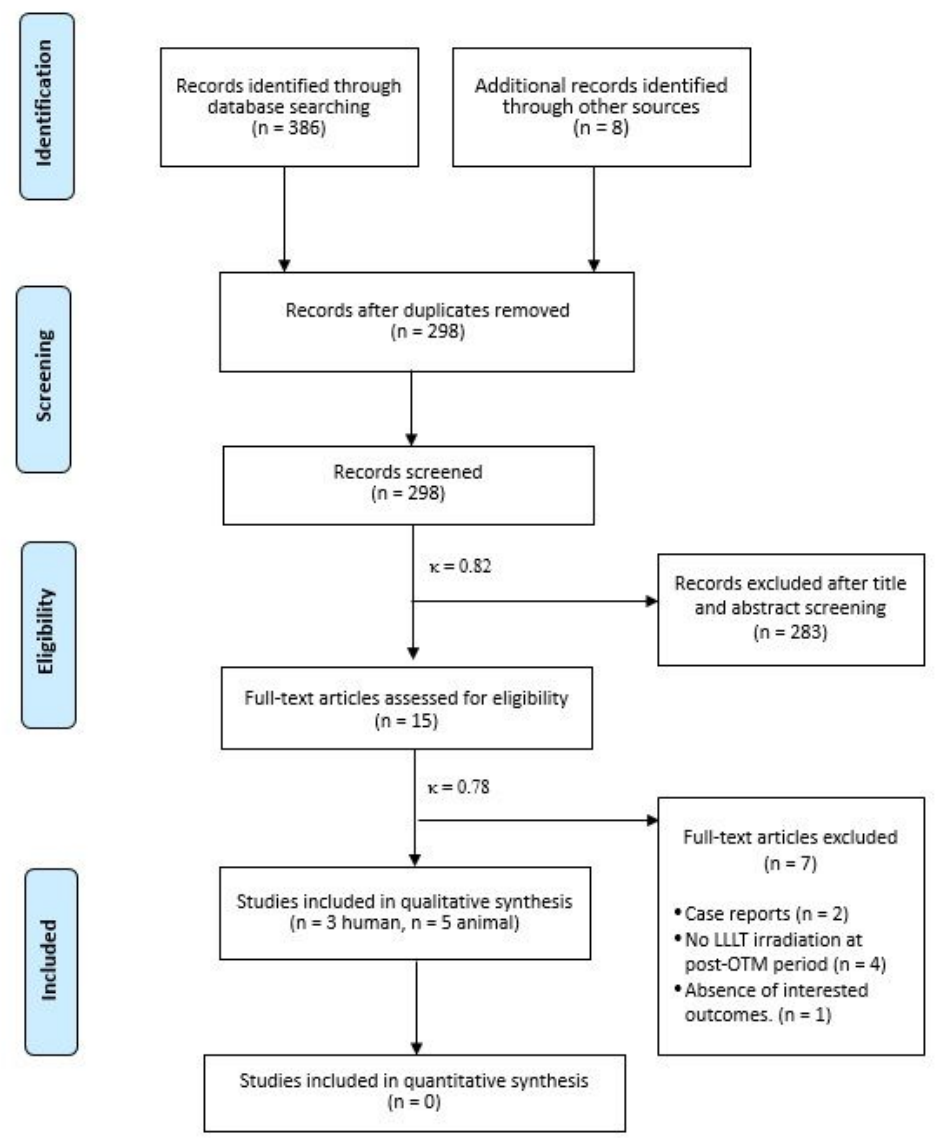

Figure 1

PRISMA flow diagram summarizing the literature search 
Risk of Bias for clinical studies

Random sequence generation (selection bias)

Allocation concealment (selection bias)

Blinding of participants and personnel (performance bias)

Blinding of outcome assessment (detection bias)

Incomplete outcome data (attrition bias)

Selective reporting (reporting bias)

Other bias

$\begin{array}{lllll}0 \% & 25 \% & 50 \% & 75 \% & 100 \%\end{array}$

Low risk of bias

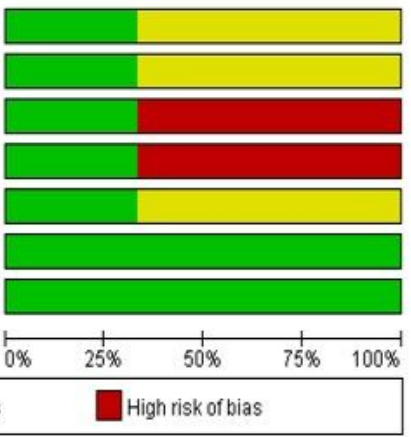

Figure 2

Assessment of risk of bias item presented as percentages across all included clinical studies Risk of Bias for animal studies

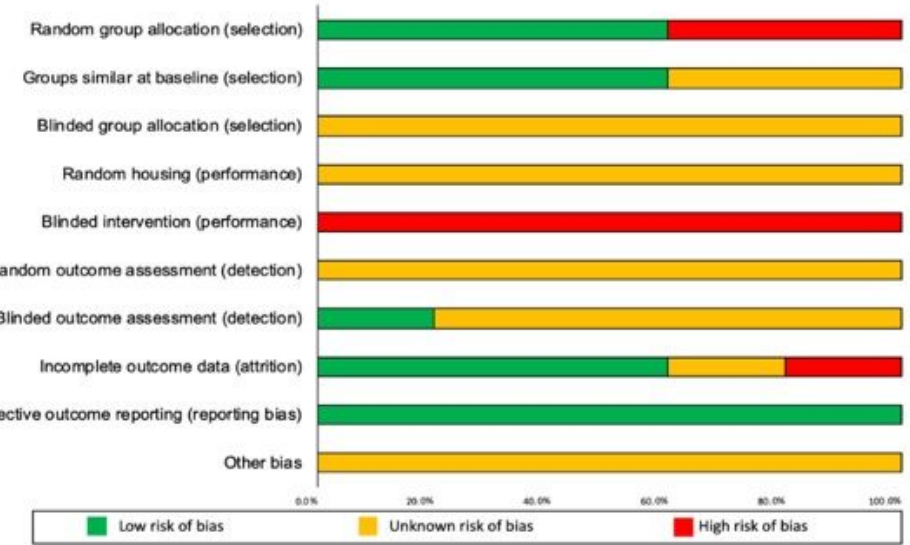

\section{Figure 3}

Assessment of risk of bias item presented as percentages across all included animal studies

\section{Supplementary Files}

This is a list of supplementary files associated with this preprint. Click to download.

- searchstrategy.docx

- conflictofinterestform.pdf

- PRISMAflowchart.docx

- PRISMAchecklist.doc 